\title{
Surgical Management of Coexisting Primary Splenic Abscess and Lymphoma: Case Report and Review of Literature
}

\author{
Sundus Abbasi ${ }^{\mathrm{a}, \mathrm{c}}$, Emma Punni ${ }^{\mathrm{a}}$, Elias Fakhoury ${ }^{\mathrm{a}}$, Patrick Michael ${ }^{\mathrm{b}}$, Hoan Bui ${ }^{\mathrm{a}}$
}

\begin{abstract}
We present an extremely rare case of combined splenic abscess and Bcell lymphoma in a patient who was initially admitted for congestive heart failure. Due to its non-specific clinical picture, the final diagnosis was challenging. The patient was initially conservatively managed with antibiotics and percutaneous drainage of the abscess and then definitively managed with a splenectomy. This is the first case described with both pathological processes present simultaneously.
\end{abstract}

Keywords: Splenic abscess; B-cell lymphoma; Splenectomy

\section{Introduction}

Isolated splenic abscess and splenic lymphoma has been diagnosed and treated with both medical and surgical treatments. However, the diagnostic sensitivity and treatment plans are not well established. We hope this paper will increase the clinical suspicion of splenic pathology in patients with similar clinical presentations.

\section{Case Report}

A 71-year-old male with no significant past medical history presented to the emergency department with worsening bilateral lower extremity swelling for 3 weeks and associated productive cough. A two-dimensional echocardiogram showed an ejection fraction of $45-50 \%$ with grade 2 diastolic dysfunction. A subsequent chest radiograph showed a right pleural effusion. Diagnostic thoracentesis confirmed an exudative effusion. A computed tomography (CT) of the chest after thoracentesis revealed a left hydropneumothorax and a complex

Manuscript accepted for publication August 19, 2016

aDepartment of Surgery, Saint Joseph's Regional Medical Center, Paterson, NJ, USA

bDepartment of Internal Medicine, Saint Joseph's Regional Medical Center, Paterson, NJ, USA

${ }^{\mathrm{c} C}$ Corresponding Author: Sundus Abbasi, Department of Surgery, Saint Joseph's Regional Medical Center, Paterson, NJ, USA.

Email: Sundus.abbasi@gmail.com subcapsular splenic collection. At this time, there was concern for iatrogenic injury or a splenic abscess. After further review, the findings were more concerning for a perisplenic and intrasplenic abscess (Fig. 1). Subsequently, the patient underwent CT-guided drainage of the subcapsular splenic abscess. Cultures from the purulent fluid confirmed many salmonella species and the patient was started on rocephin $2 \mathrm{~g}$ daily as per infectious disease recommendations. A radiographic barium enema was done to evaluate for possible etiology. At the splenic flexure, there was a single, large, and irregular diverticulum with a walled microperforation. A repeat CT of abdomen showed new splenic abscesses with interval decrease in the largest splenic abscess. Additionally, there were multiple retroperitoneal and mediastina nodes suggesting mediastinal and celiac axis nodes. The patient had failed conservative treatment and underwent a laparoscopic converted to open splenectomy revealing a densely encapsulated splenic abscess cavity and enlarged loculated spleen (Figs. 2 and 3). The patient lost $1 \mathrm{~L}$ of blood during the procedure, received 6 units of packed red blood cells, 2 units of fresh frozen plasma, 2 units of albumin, and $2.8 \mathrm{~L}$ of lactated ringers. The patient had an uncomplicated post-surgical course while in the hospital. Final histopathology of the spleen revealed diffuse large B-cell lymphoma.

\section{Discussion}

Splenic abscess is uncommon, occurring in $0.14 \%$ of patients and can be caused by hematogenous spread, penetrat-

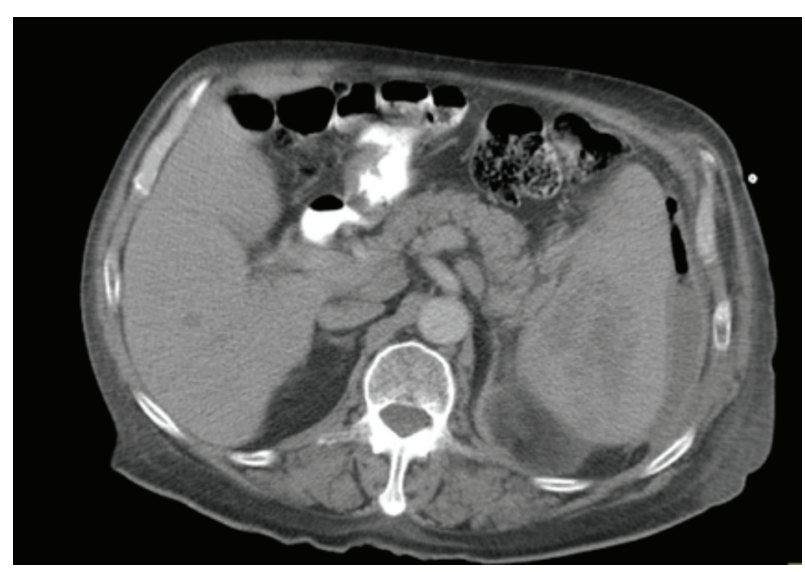

Figure 1. CT scan showing an abnormal spleen. 


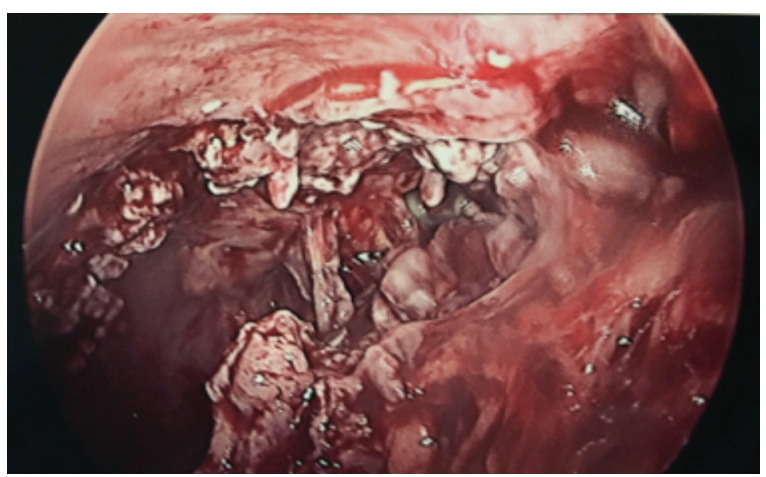

Figure 2. Intraoperative laparoscopic image of a necrotic, diseased spleen.

ing wounds, and infectious complications [1]. Splenic abscess occurs in patients with weakened immune systems including neoplastic processes, immunodeficiency, trauma, metastatic infection, splenic infarct or diabetes. While these conditions may explain a single splenic abscess, multiple splenic abscesses are rare, which, in the setting of lymphoma has not been previously reported. Documented splenic abscesses have occurred after sleeve gastrectomies postulating the cause from immunosuppression in the setting of rapid weight loss, limited oral intake and in conjunction with endocarditis [2, 3]. Around $44 \%$ of patients present with left upper quadrant pain, fever, and leukocytosis. Interestingly, as present in our patient, an abnormal chest radiograph has been noted in patients, with a left pleural effusion as the most common finding [1]. Common pathogens include Salmonella species, Streptococcus viridians and Klebsiella pneumonia $[1,4]$. Computed tomographic (CT) scanning remains the gold standard for splenic abscess. Ultrasound can also be used, approaching a sensitivity of $98 \%$ $[5]$.

Malignant lymphoma was initially diagnosed at splenectomy without evidence of peripheral disease is also rare. The spleen is involved in $30-40 \%$ of systemic lymphomas, while primary lymphoma of the spleen occurs in $<1 \%$ of patients $[6,7]$. This has been referred to as malignant lymphoma with prominent splenic involvement (LPS) or primary splenic lymphoma (PSL), incidence of both approximately 5\% [8]. Most common subtypes included diffuse large B-cell lymphoma and splenic marginal B-cell lymphoma findings are usually consistent with hypo-attenuation or iso-attenuation in plain CT. These tumors also display a large area of necrosis [8]. MRI can be used as an adjunct to imaging, but definitively PET-CT plays the most important role in staging, restaging, and therapy monitoring for lymphoma. While MRI was not used in our patient, CT findings were consistent with the diagnosis of splenic abscess (Fig. 1).

\section{Conclusion}

This is the first reported case of coexisting splenic abscesses

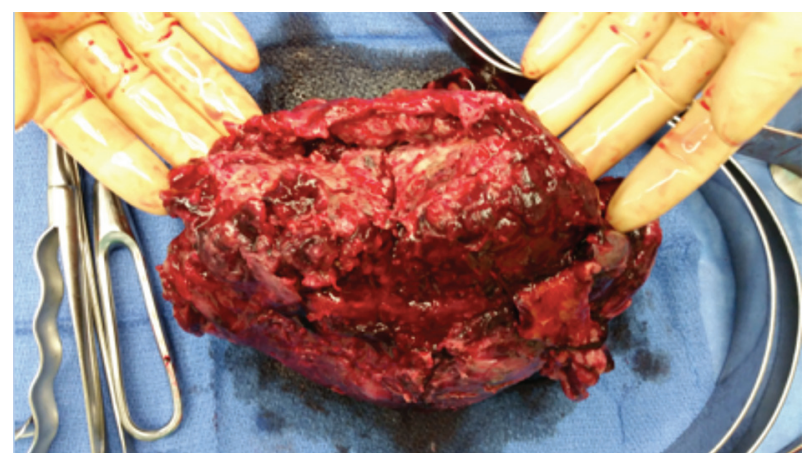

Figure 3. Intraoperative spleen after removal.

and PSL. This case report intertwines both principles of medicine with surgery. We hope this paper will help increase the diagnostic sensitivity and differential diagnosis in similar patients.

\section{Conflicts of Interest}

There were no conflicts of interest from all authors in this case report.

\section{References}

1. Lee WS, Choi ST, Kim KK. Splenic abscess: a single institution study and review of the literature. Yonsei Med J. 2011;52(2):288-292.

2. Schiavo L, Scalera G, De Sena G, Ciorra FR, Pagliano P, Barbarisi A. Nonsurgical management of multiple splenic abscesses in an obese patient that underwent laparoscopic sleeve gastrectomy: case report and review of literature. Clin Case Rep. 2015;3(10):870-874.

3. Elasfar A, AlBaradai A, AlHarfi Z, Alassal M, Ghoneim A, AlGhofaili F. Splenic abscess associated with infective endocarditis; Case series. J Saudi Heart Assoc. 2015;27(3):210-215.

4. Cabadak H, Erbay A, Karaman K, Sen S, Tezer-Tekce Y. Splenic abscess due to Salmonella enteritidis. Infect Dis Rep. 2012;4(1):e4.

5. Ooi LL, Nambiar R, Rauff A, Mack PO, Yap TL. Splenic abscess. Aust N Z J Surg. 1992;62(10):780-784.

6. Caremani M, Occhini U, Caremani A, Tacconi D, Lapini L, Accorsi A, Mazzarelli C. Focal splenic lesions: US findings. J Ultrasound. 2013;16(2):65-74.

7. Kaza RK, Azar S, Al-Hawary MM, Francis IR. Primary and secondary neoplasms of the spleen. Cancer Imaging. 2010;10:173-182.

8. Li M, Zhang L, Wu N, Huang W, Lv N. Imaging findings of primary splenic lymphoma: a review of 17 cases in which diagnosis was made at splenectomy. PLoS One. 2013;8(11):e80264. 